\title{
Neural activity with spatial and temporal correlations as a basis to simulate fMRI data
}

\author{
Marijke Welvaert \& Yves Rosseel
}

\section{Introduction}

In the development of data analysis techniques, simulation studies are constantly gaining more interest. The largest challenge in setting up a simulation study is to create realistic data. This is especially true for generating fMRI data, since there is no consensus about the biological and physical relationships underlying the BOLD signal. Most existing simulation studies start from empirically acquired resting data to obtain realistic noise and add known activity (e.g., Bianciardi et al., 2004). However, since you have no control over the noise, it is hard to use these kinds of data in simulation studies. Others use the Bloch equations to simulate fMRI data (e.g., Drobnjak et al., 2006). Even though they get realistic data, this process is very slow involving a lot of calculations which might be unnecessary in a simulation study. We propose a new basis for generating fMRI data starting from a neural activation map where the neural activity is correlated between different locations, both spatial and temporal. A biologically inspired model can then be used to simulate the BOLD response.

\section{Methods}

We chose to represent the fMRI image by a grid. Coordinates were chosen such that the unit (e.g., voxel) which was activated by a stimulus, acted as the center of the coordinate system (i.e. $(0,0)$ in a twodimensional grid). Moving away from the activated unit in the grid resulted in a decreasing neuronal response with a temporal delay defined by the rate $\rho$. For each unit, the haemodynamic response was calculated according to the model as described in Buxton et al. (2004).
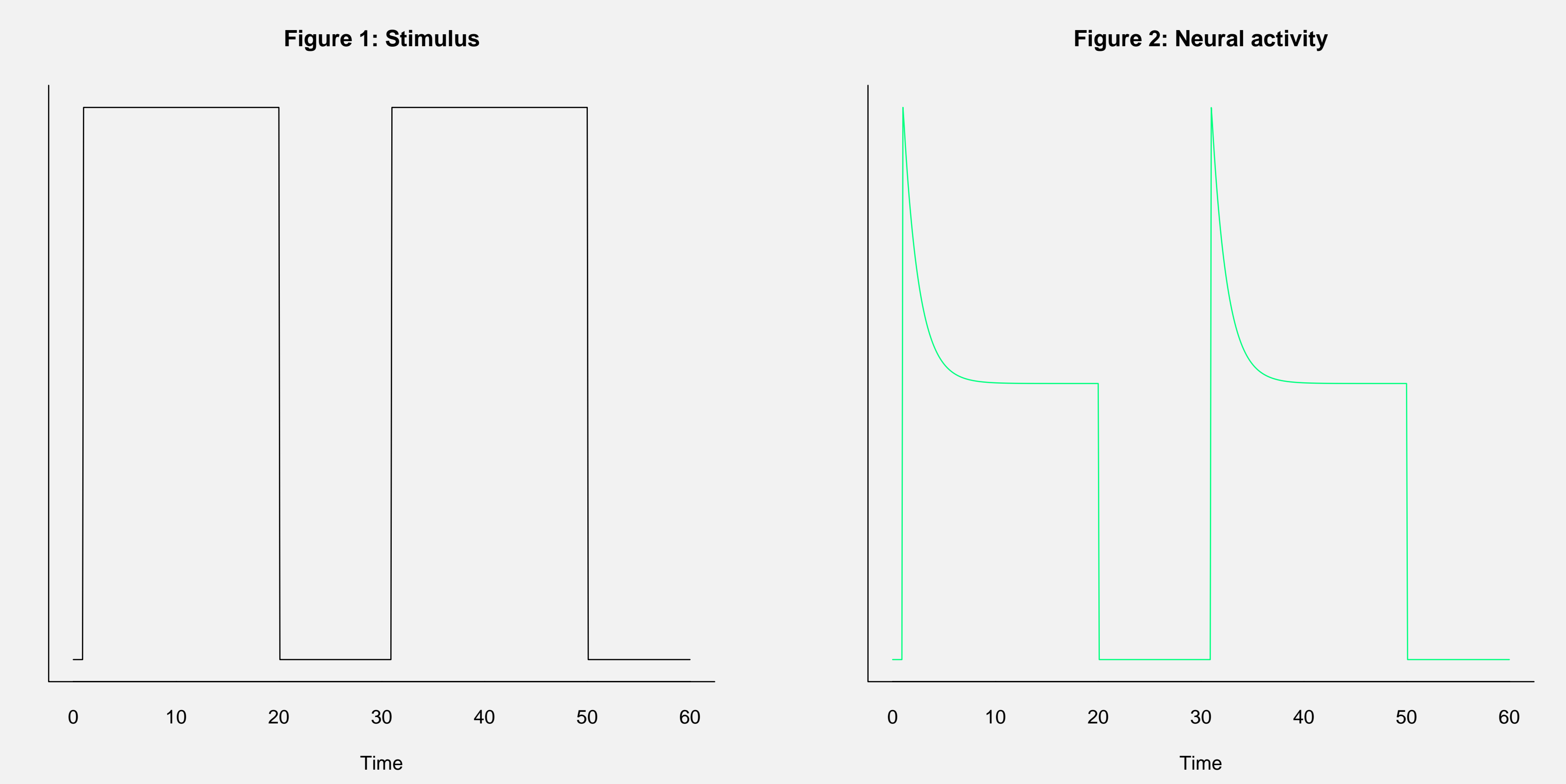

Neural activity was modelled as the difference between an excitatory stimulus function and an inhibitory function. Consequently, the neural response to a sustained stimulus is an initial peak followed by a decay to a lower plateau level.
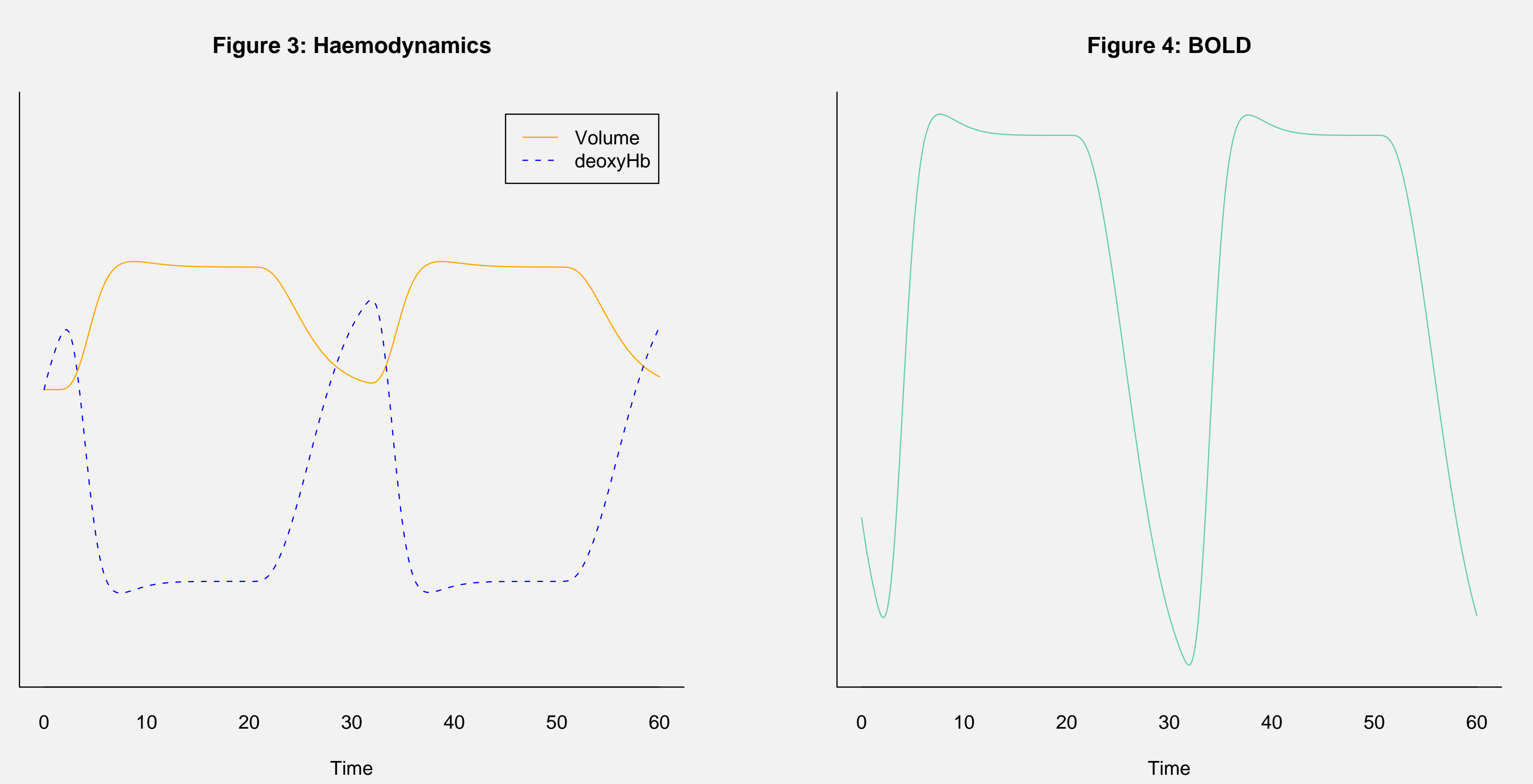

Neurovascular coupling of the neural response results in $\mathrm{CBF}$ and $\mathrm{CMRO}_{2}$ responses entering the balloon model. The output of the balloon model, cerebral blood volume and deoxygenated haemoglobin responses, are then combined in the BOLD response.
Results

Neural activity and BOLD response resulting from two blocks of a 20 s sustained activation of a unit in a 10 by 10 two-dimensional grid are presented in Figure 5 and 6 . It can be seen that the neural activity spreads out in both space and time. Correspondingly, the BOLD response in the activated unit is larger and peaks earlier compared to surrounding units.

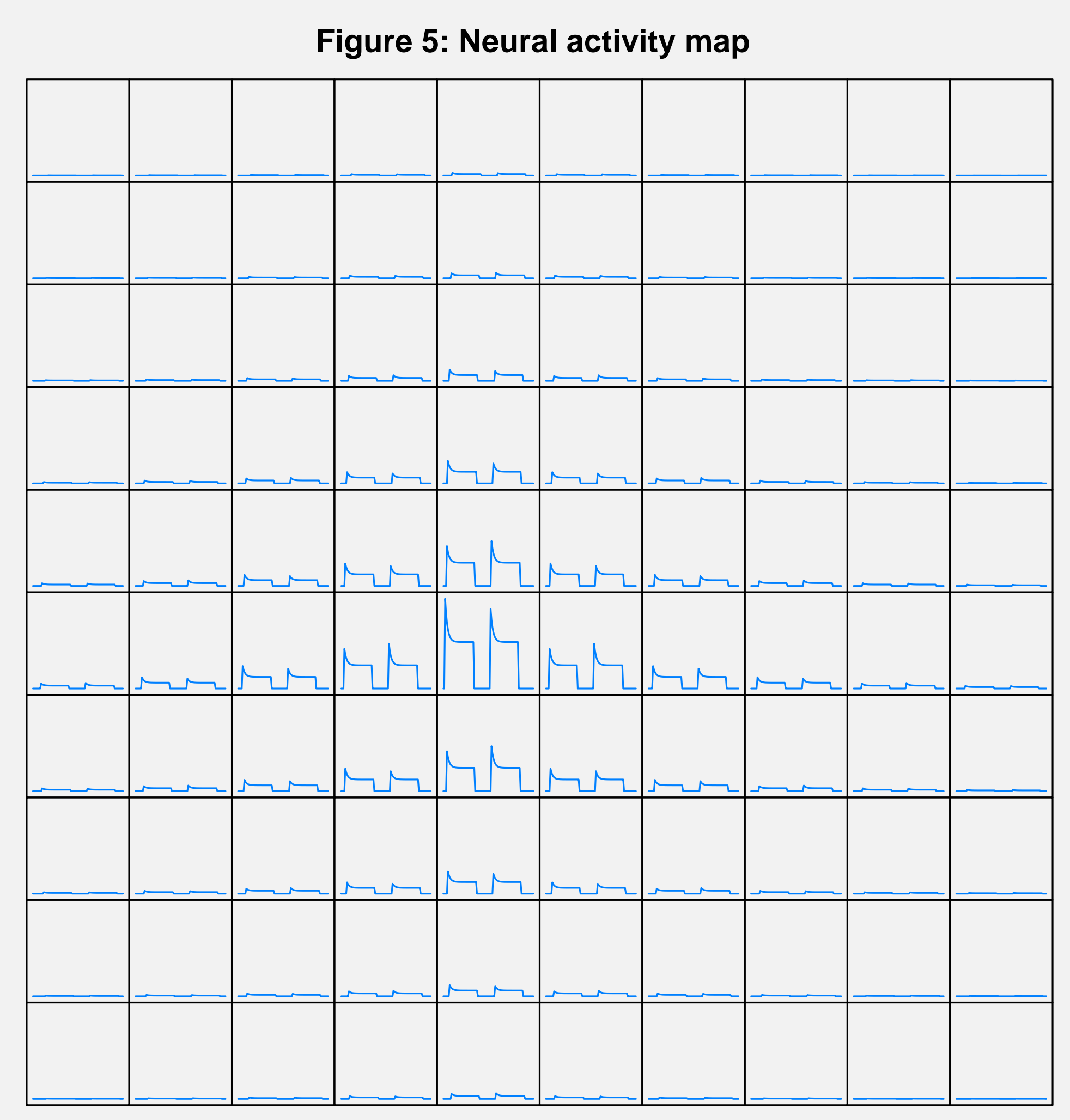

This results in an activated region in the grid where the center is more activated than the boundaries. Extension to a more realistic situation with several stimulus blocks which activate multiple regions in a threedimensional brain is straightforward.

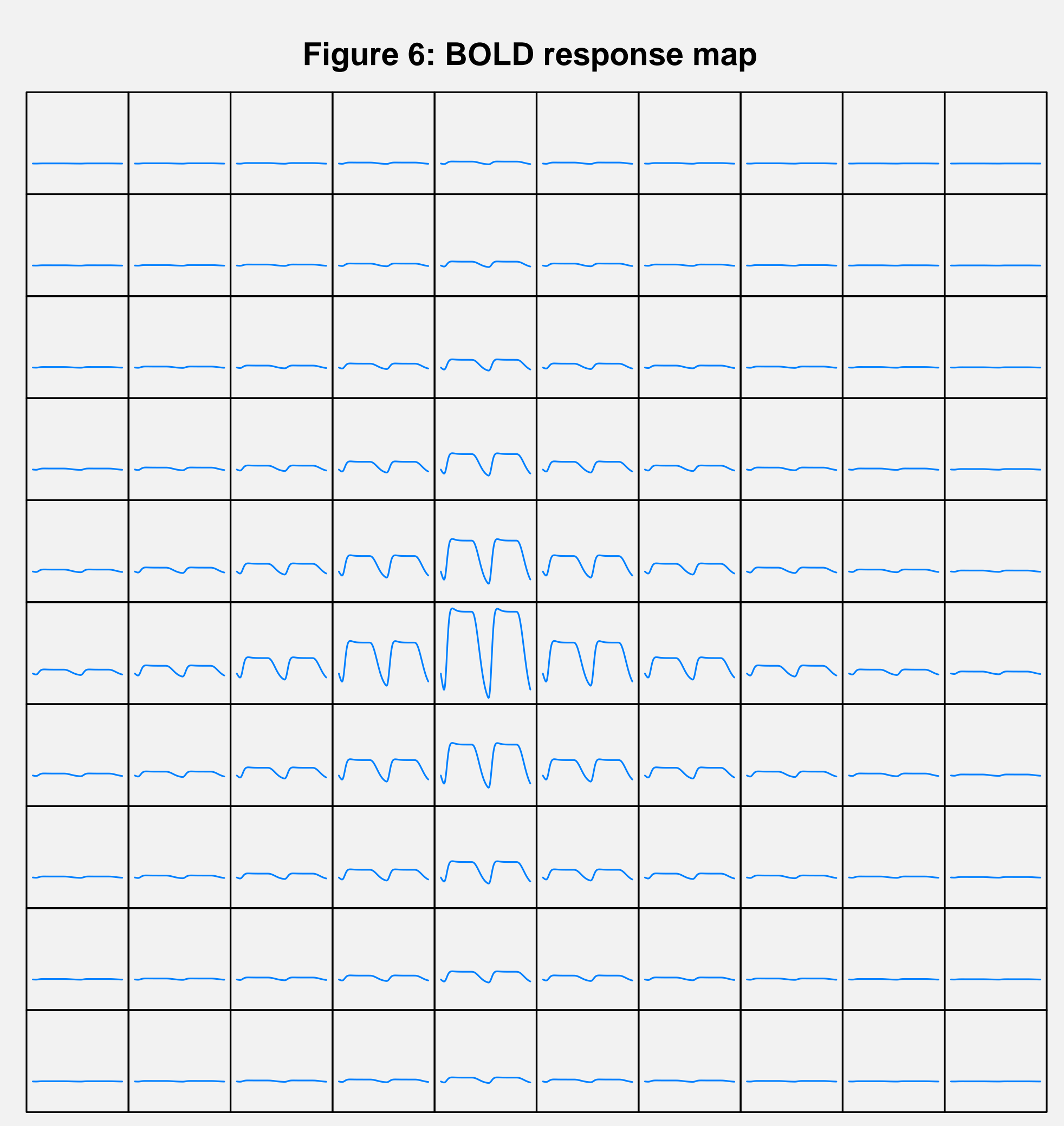

\section{Conclusions}

The advantage of the proposed neural activity map as compared to simulating ordinary time series is that we take into account the spatial and temporal correlations of the BOLD response early in the process. Therefore, this map can be considered as a model of the working brain which can be used as a basis for simulating realistic fMRI data.

\section{References}

Bianciardi, M., Cerasa, A., Patria, F., \& Hagberg, G.E. (2004), 'Evaluation of mixed effects in eventrelated fMRI studies: impact of first-level design and filtering', NeuroImage, vol. 22, no. 3, pp. 1351-1370.

Buxton, R.B, Uludağ, K., Dubowitz, D.H., \& Liu, T.T. (2004), 'Modeling the hemodynamic response to brain activation', NeuroImage, vol. 23, no. 1, pp. S220-S233.

Drobjnak, I., Gavaghan D., Süli, E., Pitt-Francis, J., \& Jenkinson, M. (2006), 'Development of a fMRI simulator for modelling realistic rigid-body motion artifacts', Magnetic Resonance in Medicine, vol. 56, no. 2, pp. 364-380. 